\title{
Associative Encoding and Retrieval Are Predicted by Functional Connectivity in Distinct Hippocampal Area CA1 Pathways
}

\author{
Katherine Duncan, ${ }^{1}$ Alexa Tompary, ${ }^{2}$ and Lila Davachi ${ }^{2,3}$ \\ ${ }^{1}$ Department of Psychology, Columbia University, New York, New York 10027 and ${ }^{2}$ Department of Psychology and ${ }^{3}$ Center for Neural Science, New York \\ University, New York, New York 10003
}

\begin{abstract}
Determining how the hippocampus supports the unique demands of memory encoding and retrieval is fundamental for understanding the biological basis of episodic memory. One possibility proposed by theoretical models is that the distinct computational demands of encoding and retrieval are accommodated by shifts in the functional interaction between the hippocampal CA1 subregion and its input structures. However, empirical tests of this hypothesis are lacking. To test this in humans, we used high-resolution fMRI to measure functional connectivity between hippocampal area $\mathrm{CA} 1$ and regions of the medial temporal lobe and midbrain during extended blocks of associative encoding and retrieval tasks. We found evidence for a double dissociation between the pathways supporting successful encoding and retrieval. Specifically, during the associative encoding task, but not the retrieval task, functional connectivity only between area CA1 and the ventral tegmental area predicted associative long-term memory. In contrast, connectivity between area CA1 and DG/CA3 was greater, on average, during the retrieval task compared with the encoding task, and, importantly, the strength of this connectivity significantly correlated with retrieval success. Together, these findings serve as an important first step toward understanding how the demands of fundamental memory processes may be met by changes in the relative strength of connectivity within hippocampal pathways.
\end{abstract}

Key words: CA1; CA3; functional connectivity; high-resolution fMRI; hippocampus; VTA

\section{Introduction}

In everyday experience, memory encoding and retrieval appear seamlessly intertwined; encoding provides material that will later be retrieved, whereas retrieval supplies context to facilitate meaningful encoding. However, the neural mechanisms thought to underlie these processes have been hypothesized to place opposing demands on the episodic memory system (Marr, 1971; O'Reilly and McClelland, 1994). Specifically, the rapid formation of memory traces representing single episodes requires high levels of synaptic plasticity that, during retrieval, could lead to the overwriting of stored memories. Conversely, successful associative retrieval requires that cues reactivate related memory traces and thus benefits from a low threshold for memory reinstatement, but reinstating related memories at the time of encoding could lead to proactive interference (O'Reilly and McClelland, 1994).

Although it is generally agreed that the hippocampus is critical for both encoding and retrieval (Scoville and Milner, 1957;

Received Feb. 5, 2014; revised May 21, 2014; accepted June 26, 2014.

Author contributions: K.D. and L.D. designed research; K.D. performed research; K.D. and A.T. analyzed data; K.D., A.T., and L.D. wrote the paper.

This work was supported by National Institute of Mental Health Grant MH074692 (L.D.). K.D. was supported by a Canadian Institutes of Health Research Fellowship.

Correspondence should be addressed to Lila Davachi, Department of Psychology, New York University, 6 Washington Place, 8th Floor, New York, NY 10003. E-mail: lila.davachi@nyu.edu.

DOI:10.1523/JNEUROSCI.0521-14.2014

Copyright $\odot 2014$ the authors $\quad 0270-6474 / 14 / 3411188-11 \$ 15.00 / 0$
Eichenbaum et al., 2007; Squire et al., 2007), it remains unknown how the hippocampus supports the complex demands of both processes. However, the unique pattern of afferent projections converging on area CA1, the major hippocampal output subregion, may provide an answer. On the one hand, inputs to CA1 might differentially support long-term memory encoding. Specifically, both late-phase long-term potentiation (LTP) at CA1 synapses and long-term memory formation have been demonstrated to be dopamine dependent (Frey et al., 1990; Huang and Kandel, 1995; Li et al., 2003; O'Carroll et al., 2006). Thus, the formation of long-lasting memories may be facilitated during periods of increased connectivity between area CA1 and the ventral tegmental area (VTA), its primary dopaminergic afferent (Gasbarri et al., 1997). Furthermore, the processing of sensory representations during encoding is thought to be prioritized via enhanced connectivity between CAl and the medial temporal lobe cortical regions that carry this information (Hasselmo et al., 2002; Colgin and Moser, 2010; Giraud et al., 2011; Duncan et al., 2012b).

On the other hand, CA1 is also the primary output pathway for area CA3, a region thought to support associative retrieval (Marr, 1971; Treves and Rolls, 1992). This pathway serves as a critical gate through which information from area CA3 must pass before propagating into cortical representational areas (McClelland et al., 1995). Thus, associative retrieval may be specifically facilitated during periods of high connectivity between areas CA3 
Day 1 (scanned)
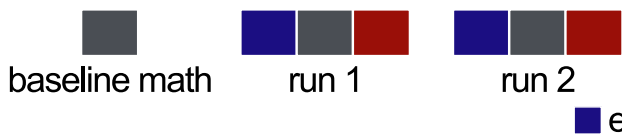

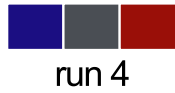

retrieval
Day 2 (not scanned)

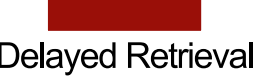

b

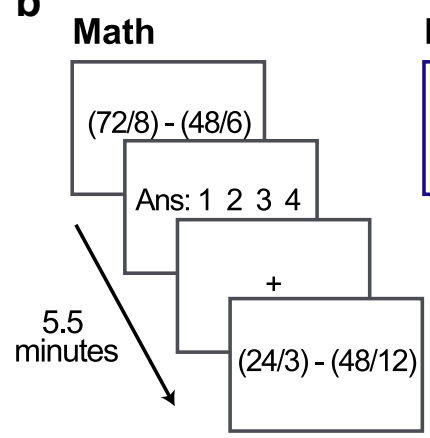

Encoding

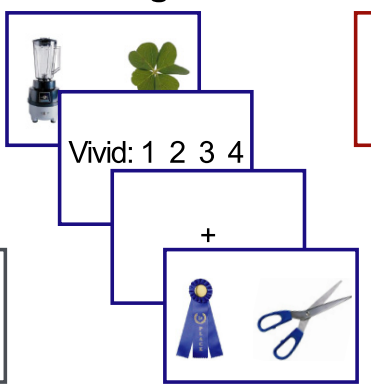

Retrieval

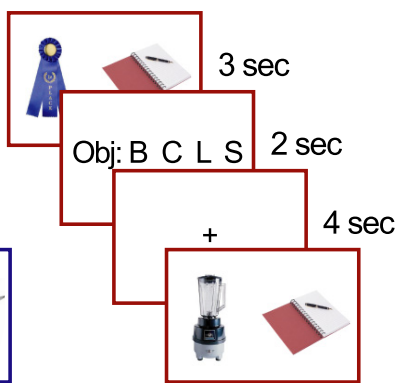

Delayed Retrieval

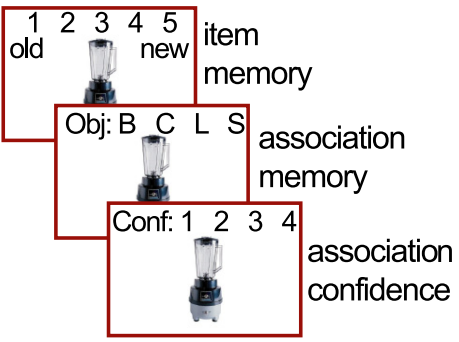

Figure 1. Experimental design. $\boldsymbol{a}$, Schematic illustration of session structure. The experiment was conducted over $2 \mathrm{~d}$. The first day, which was scanned, began with a baseline math block followed by four cycles of encoding, math, and retrieval blocks. Each block was 5.5 min long. Participants returned the following day for the un-scanned Delayed Retrieval test. $\boldsymbol{b}$, Example trials for each task. In the Math Task, participants answered arithmetic problems. The solution was always 1,2,3, or 4. In the Encoding Task, participants formed mental images of two objects interacting. The left object was always unique to the trial, and the right one was selected from a set of four repeating objects. Participants rated the vividness of their imagery on a scale from 1 to 4 . In the Retrieval Task, participants recalled which of the four repeated objects was paired with the object on the left side of the screen. The notebook image on the right side of the screen served as a placeholder for the target object. The Delayed Retrieval Task had three steps. First, participants indicated whether an object was studied the day before or if it was new (item memory). Next, if the participants thought the object was old, they were asked whether they could remember which repeated object was paired with it (associative memory). Finally, if they recalled the associated object, they were asked how confident they were on a scale from 1 to 4 . In all tasks, participants were given the option to indicate that they were unsuccessful rather than guessing.

and CA1 (Hasselmo et al., 2002; Montgomery and Buzsaki, 2007; Colgin and Moser, 2010; Duncan et al., 2012b).

Together, this work suggests that dynamic alterations in the strength of CA1 connectivity may be important in biasing processing toward encoding or retrieval. However, to our knowledge, no one has examined whether CAl connectivity is differentially related to encoding and retrieval operations. To address this question, participants engaged in interleaved blocks of associative encoding and retrieval while undergoing highresolution fMRI. CA1 functional connectivity was measured during extended blocks of encoding and retrieval tasks to determine (1) whether connectivity was significantly modulated by memory task and (2) whether changes in CA1 functional connectivity were related to behavioral measures of encoding and retrieval success.

\section{Materials and Methods}

Participants

Seventeen (eight female) right-handed members of the New York University community with normal or corrected-to-normal vision participated in the study. One subject was removed from the analysis because of experimental error. The remaining 16 participants had a mean age of 27.1 years, with a range of 22-35 years. All experimental procedures were approved by the Institutional Review Board at New York University, and informed consent was obtained for each participant at the beginning of the experimental session.

\section{Behavioral procedures}

Participants performed an associative memory task that was specifically designed to focus mnemonic processing on either episodic encoding or retrieval for extended periods of time. Each session began with a $5.5 \mathrm{~min}$ block of the Baseline Math Task. Next, participants completed four scans, each of which began with a 5.5 min block of the Encoding Task, followed by a 5.5 min block of the Math Task, and ended with a 5.5 min block of the Retrieval Task. Each Retrieval Task tested memory for the associations presented during that block's preceding Encoding Task, making the retention period $11 \mathrm{~min}$, on average. All three tasks (Encoding, Math, and Retrieval) were similarly structured with identical timing, the same number of response options, and similar levels of difficulty as assessed by accuracy (Fig. 1). Participants returned $\sim 24 \mathrm{~h}$ after the scanning session and completed a surprise long-term memory test.

Encoding Task. Each trial began with the presentation of a pair of objects for 3 s. Participants were asked to spend this entire period creating and elaborating on a mental image of the two objects interacting. The object on the left side of the screen was unique on every trial, whereas the object on the right side of the screen was selected from a set of four repeating objects (baby bottle, clover, lobster, scissors). Next, a vividness-rating screen was presented for $2 \mathrm{~s}$. The scale ranged from 1 to 4 and was counterbalanced across participants (e.g., 4 corresponded to most vivid for half of the participants and least vivid for the other half). Responses were made using the finger keys on an MRI-compatible button box. Participants also had the option to indicate that they were unsuccessful in forming an image using the thumb key. The rating period was followed by a $4 \mathrm{~s}$ fixation period. The fixation cross turned from black to red for the last $1 \mathrm{~s}$ of the fixation period so that participants could prepare for the presentation of the next pair of objects. Each scanned Encoding Task block included 36 trials. Each of the four repeating associates was presented in nine trials per block.

Before the scanned session, participants were informed that their memory would be tested during the Retrieval Task blocks. They were provided with two examples of scenarios in which an example pair of objects interacted and one example in which the two objects did not interact with each other. They were strongly encouraged to generate scenarios that were creative and vivid and that maximized the interaction between objects, being told that this would improve their ability to recall the associations during the Retrieval Task. After the instructions, participants completed a practice block of Encoding, Math, and Retrieval Tasks (six trials each).

Retrieval Task. The Retrieval Task was designed to tax participants' associative retrieval while minimizing the presentation of stimulus novelty to reduce any automatic encoding processes. To that end, participants were re-presented with the trial-unique objects studied during the preceding Encoding Task and were asked to recall which of the four repeated associates was paired with them. Each trial began with the presentation of the previously studied object on the left side of the screen and 
a placeholder image of a notebook (a familiar image that was used during practice trials) on the right side of the screen. The placeholder image was simply used to make the visual displays more similar during the Encoding and Retrieval Tasks. Participants were asked to spend the full $3 \mathrm{~s}$ of stimulus presentation trying to determine which of the four associates were originally paired with the presented object. They were encouraged to recall the details of the scenario that they had generated so that they could be confident in their responses. Participants made their response during the following $2 \mathrm{~s}$ response period. Each of the four associates was assigned a different key. To make the response mapping intuitive, the associates were ordered alphabetically (bottle, clover, lobster, scissors), and the first letter of each object was presented in order on the response screen ("B C L S") as a reminder. Participants also began the practice session learning which key corresponded to which object. Participants had the option to indicate that they did not successfully retrieve the associate by using the thumb key and were asked not to guess. Specifically, they were told that "if you find yourself trying to decide between multiple associates, simply use the thumb key." A 4 s fixation period, identical to the fixation period in the Encoding Task, followed the response period.

Baseline Math Task. Between encoding and retrieval blocks, participants also performed a Math Task. This was chosen as a baseline task because it was similarly challenging without placing demands on the episodic memory system. During the Math Task blocks, participants solved two-part arithmetic problems [e.g., (72/8) - (24/4)] on each trial. The problems were always the subtraction of two dividends. For each trial, a problem was randomly generated with the requirement that the divisor of the division portions be no greater than 12 and that the final answer be 1, 2, 3, or 4 . The problem was displayed for $3 \mathrm{~s}$ during which participants were instructed to try to solve the problem. This was followed by a $2 \mathrm{~s}$ response period during which the participants indicated their answer $(1,2,3$, or 4$)$ using the finger keys on the button box. As was the case for the other tasks, participants had the option to indicate that they did not successfully solve the problem using their thumb key. The instructions emphasized that there was no need to guess and that it was better to accurately indicate when they were not able to perform the task than to try to increase their accuracy by guessing. After the response period, there was a $4 \mathrm{~s}$ fixation period that was identical to the fixation periods of the other tasks.

Day 2 memory test. Participants returned $\sim 24 \mathrm{~h}$ after the scanned session and performed a surprise memory test. This follow-up test was a critical aspect of the design since it allowed for the assessment of longterm memory in addition to immediate retrieval success on day 1 . On day 1 , the short retention delay and deep encoding strategies used resulted in high memory performance. This high performance was desired because it meant that estimates of functional connectivity obtained during the immediate Retrieval Task would reflect successful associative reactivation. However, the long-term memory test served an important complementary role as a more sensitive measure of the durability of those associations formed during the Encoding Task, because the $24 \mathrm{~h}$ delay allowed the weaker associations to fade. Furthermore, a prime objective of this experiment was to determine whether CA1-VTA functional connectivity is related to memory formation, and pharmacological studies in rodents (O'Carroll et al., 2006; Bethus et al., 2010) suggest that this relationship would be best identified using long-delay memory tests.

A three-step memory decision was used on day 2. Participants were presented with an object that was either studied on day 1 or was novel. They were first asked whether the object was "definitely old," "probably old," "probably new," or "definitely new." They were instructed to reserve "definitely old" responses for trials that they remembered associated details for. They were also given the option to respond "don't know" when they were uncertain. If they reported that the object was either "probably old" or "definitely old," they were next asked which of the four repeated associates was paired with it. Again, they were given the option to indicate "don't know" when they were unsure. Finally, if they reported that they remembered the associated object, they were asked how confident they were on a scale from 1 to 4 . This scale was later compressed to a high-confidence (HC) rating ( 3 and 4 ) and low-confidence (LC) rating ( 1 and 2) to increase the number of trials in the confidence bins.

\section{MRI methods}

All scanning was performed using a 3T Siemens Allegra MRI system with a whole-head coil. Functional data were collected using a zoomed highresolution echo-planar pulse (EPI) sequence similar to that used by Olman et al. (2009; TR, 1500 ms; TE, 22 ms; FOV, $192 \times 96$; 21 interleaved slices; $1.5 \times 1.5 \times 3 \mathrm{~mm}$ voxel size; flip angle, $\left.77^{\circ}\right)$. The oblique coronal slices were aligned perpendicular to the hippocampal long axis. The field of view was reduced in the phase-encode direction to reduce the total readout time and thus minimize distortions and artifacts. Saturation bands were used to suppress signal for tissue superior and inferior to the region of interest (ROI). Two high-resolution scans were collected for anatomical visualization. A T2-weighted 2D image (TR, $5100 \mathrm{~ms}$; TE, $88 \mathrm{~ms} ; 0.898 \times 0.898 \times 1.5 \mathrm{~mm}$ voxel size) was acquired in-plane to the function images. A T1-weighted high-resolution MPRAGE (magnetization-prepared rapid-acquisition gradient echo) sequence $(1 \times 1 \times 1$ voxel size; 176 sagittal slices) was also acquired to obtain full brain coverage. Finally, a field map sequence was collected to obtain estimates of the magnetic field and an in-plane spin-density image. Visual stimuli were projected onto a screen that was viewed through a mirror attached to the subject's head coil.

Preprocessing of functional data was conducted with FSL (FMRIB Software Library; available online at http://www.fmrib.ox.ac.uk/fsl) and AFNI (available online at http://afni.nimh.nih.gov/afni). The first 11 volumes (16 s) were discarded to allow for signal normalization, and then FSL's slice-timer function was used to correct for differences in slice acquisition timing. Next, the EPI volumes were motion corrected and aligned to anatomical scans using AFNI in a multistep procedure where both functional and anatomical images were coregistered to target images that were generated by the field map sequence. Functional images were first aligned within run and then were aligned to a common target across runs.

Separate anatomical ROIs were drawn for each participant's hippocampal subfields [area DG/CA2/CA3, area CA1, and subiculum (Sub)], medial temporal lobe (MTL) cortical regions (entorhinal cortex, perirhinal cortex, and parahippocampal cortex), and dopaminergic midbrain structures (VTA and substantia nigra). It should be noted that data from the entorhinal cortex were excluded from analyses because this area suffered from dropout or distortions in several participants because of its proximity to sinuses and susceptibility to distortions (Ojemann et al., 1997; Olman et al., 2009). Nearly one-third of voxels in this region suffered from dropout in the group, with one-third of participants having voxel dropout rates $>40 \%$ and two-thirds of participants having $>30 \%$ dropout.

A single observer (K.D.) drew ROIs for all participants. Hippocampal ROIs were drawn using a similar procedure to that used by Kirwan et al. (2007). The coronal plates from the study by Duvernoy (2005) were matched to the most similar coronal slice in each hippocampus and used as templates. Then, the remaining slices were filled in to form continuous regions. MTL cortical regions were drawn using the guidelines discussed by Insausti et al. (1998) and Pruessner et al. (2002). MTL regions were drawn while viewing slices from each plane on both the T1- and T2weighted EPI-aligned anatomical images to ensure that the drawing respected all anatomical boundaries. Midbrain regions were drawn on axial slices of the T2-weighted anatomical image using guidelines presented by D'Ardenne et al. (2008). All ROIs were resampled to functional resolution and masked to remove voxels that had substantial dropout or distortions in the EPI images, identified with a combination of visual examination and EPI masks generated with the 3dAutomask function (available online at http://afni.nimh.nih.gov/afni).

\section{fMRI statistical analysis}

State-based background connectivity. There is mounting evidence that episodic encoding and retrieval can be influenced by processes unfolding before and after an encoding or retrieval opportunity (Tambini et al., 2010; Addante et al., 2011; Ben-Yakov and Dudai, 2011; Fell et al., 2011; Duncan et al., 2012b). In one notable example, novelty-triggered release of dopamine in the hippocampus enhanced LTP for several minutes after the animal was removed from the novel environment (Li et al., 2003). 
Thus, the relationship between memory performance and hippocampal network functional connectivity can be, and may even best be, assessed at a broader time scale than individual trials. Therefore, we designed the experiment to include long blocks of the Encoding and Retrieval Tasks so that we could measure background connectivity over these more extended time frames and relate this measure of connectivity to encoding and retrieval performance during those same blocks. This type of approach has recently been used in the attention and working memory domains to demonstrate the functional relevance of background connectivity (Newton et al., 2011; Norman-Haignere et al., 2012).

With this motivation, participants performed alternating blocks of trials that consisted of encoding novel associations and subsequently retrieving them. A block duration of $5.5 \mathrm{~min}$ was selected based on the optimization of resting-state functional connectivity analyses (Van Dijk et al., 2010). We quantified task (block)-dependent changes in CA1 background connectivity across entire blocks of Encoding and Retrieval Tasks. This measure of connectivity is, thus, sensitive to coordinated processing leading up to, during, and after a trial and can be thought of as a measure of connectivity that likely reflects state-based changes. Estimates obtained using this type of approach, however, could be inflated by common sources of noise, or in situations where pairs of regions show similar, but independently computed, responses to trials based on experimental conditions. Thus, we removed several nuisance factors before this calculation. First, a voxel-wise GLM was conducted using AFNI's $3 \mathrm{dDeconvolve} \mathrm{that} \mathrm{included} \mathrm{regressors} \mathrm{for} \mathrm{nuisance} \mathrm{factors} \mathrm{and} \mathrm{task-}$ evoked responses. The nuisance regressors included (1) the six motion parameters calculated during motion correction, (2) the mean signal from white matter ROIs, and (3) the mean signal from ventricular ROIs. It should be noted that midbrain regions are particularly susceptible to physiological noise in the BOLD signal, such as respiration and heart rate, because of their close proximity to large vasculature. The inclusion of these nuisance factors, along with temporal filtering described below, can reduce but will not eliminate this source of noise (Birn et al., 2006). The trial-evoked regressors were included along with their temporal and dispersion derivatives to flexibly reduce the influence of trial-evoked responses. These regressors were generated by binning trials according to accuracy and convolving $3 \mathrm{~s}$ boxcars, corresponding to trial periods, with a hemodynamic response function (HRF; see below, GLM for trialevoked responses, for additional details). Next, the residuals from this model, which reflect fluctuations in the BOLD response that are not tied to a particular type of event, were bandpass filtered into the $0.01-0.1 \mathrm{~Hz}$ range using AFNI's 3dBandpass function. This frequency band is commonly used when calculating correlations during resting-state scans (Cordes et al., 2001) as well as during extended task blocks (Newton et al., 2011) because it minimizes the effects of high- and low-frequency scanner noise. This filtering also removed run-related differences in signal intensity, allowing for later concatenation of signal across the four runs.

Critically, the temporal filtering also served as a second means of removing trial-evoked responses. All three tasks were designed to have the same periodic timing: one trial every $9 \mathrm{~s}(0.111 \mathrm{~Hz})$. Because the task frequency is higher than the upper bound of the bandpassed filter, the filter removed any responses consistently elicited by trials within a block without making any assumptions about the shape or timing of the HRF in a voxel. Moreover, the use of a periodic design does not introduce any systematic biases across tasks because the timing of trial presentation was identical in all tasks. Later estimation of differences between conditions of interest (e.g., correct vs incorrect performance) was possible without temporal jittering, since the ordering of trials across conditions was randomized because of subject performance (Burock et al., 1998).

After removal of these nuisance factors, the average time series within each ROI was calculated, and a regression was used to compute background functional connectivity between ROIs during each of the three tasks (Encoding, Retrieval, and Math). First, each run was divided into the time points that corresponded to the Encoding (volumes 1-217), Math (volumes 223-439), and Retrieval (volumes 445-661) Tasks. The 217 time points collected during the Baseline Math scan were also separately extracted. Next, for each region, the average signal for each task was concatenated across runs. Three participants reported difficulties staying awake during a subset of their scans (one participant had difficulty on one run, and the remaining two had difficulty on two runs). Performance on these runs was $>2$ SD below the group's mean, confirming the participants' reports, so these runs were removed from all analyses.

To compute connectivity between area CA1 and other regions, we used a regression to predict the average CA1 time series with the time series extracted from the other regions, separately for each task and each subject. Thus, each regression analysis resulted in $\beta$ estimates that reflect the degree to which the signal in each ROI predicted the signal in area CA1 during the Encoding or Retrieval Tasks for each subject. These $\beta$ estimates were then entered into a repeated-measures ANOVA that included predictor region and task as factors. Finally, $t$ tests were used to measure simple effects of task for individual predictor regions. Additionally, in a control analysis, we adjusted for the order of Encoding and Retrieval Task scans by restricting our analysis to a subset of runs (Encoding, runs 2-4; Retrieval, runs 1-3).

Memory correlations with background connectivity. To quantify the relationship between background connectivity and memory performance, we correlated background connectivity with memory performance across subjects. First, baseline functional connectivity (Baseline Math) was subtracted from connectivity during each of the task periods. Next, behavioral measures of successful encoding and retrieval were calculated. Successful long-term associative memory performance was assessed by computing a corrected high-confidence associative memory score for each subject [day 2, High-Confidence Correct - (High-Confidence Incorrect)/4], and retrieval success was computed using a correctedmemory score for performance during the Retrieval Task [day 1, Source Correct - (Source Incorrect)/4]. The relationship between long-term memory performance (day 2) and CA1 background connectivity during the Encoding Task was then computed to assess the formation of longlasting associative memories. Likewise, the relationship between CA1 background connectivity during the Retrieval Task and retrieval success (day 1) was also computed. The three participants who reported falling asleep during a subset of runs were not included in these analyses to avoid categorical difference in the relationship between behavior and background connectivity that could be related to sleeping.

A nonparametric shuffle approach was used to test for an interaction between region-pair and memory task (Encoding vs Retrieval). To develop a null distribution, we randomly shuffled behavioral performance and recalculated the correlations with background connectivity. Then, we calculated an interaction term [(Pair A Encoding Correlation - Pair A Retrieval Correlation) - (Pair B Encoding Correlation - Pair B Retrieval Correlation)]. We repeated this procedure 100,000 times to develop a null distribution of the interaction term. Finally, we calculated the likelihood of obtaining an interaction term as large as the one found in our experiment (two-tailed) by calculating the proportion of shuffles that resulted in an interaction term with an absolute value as large as was observed in the dataset.

We assessed the robustness of the behavioral correlations with two additional analyses. First, we replicated each analysis using robust regression, an approach that down-weights extreme, influential points. Second, we used a cross-validation procedure to predict each participant's performance based on the relationship between CA1 background connectivity and performance in the remaining participants. Separate regressions were run to predict day 1 and day 2 performance for each subject. First, the subject of interest was removed from the dataset. Then, a regression predicting day 1 corrected memory from CA1-DG/CA3 connectivity during the Retrieval Task across subjects was performed, and the resulting regression equation was used to predict the removed subject's day 1 accuracy on the basis of the CA1-DG/CA3 connectivity. Similarly, a regression that predicted day 2 corrected associative memory scores from CA1-VTA connectivity during the Encoding Task across subjects was used to predict long-term memory performance in the removed subject. The accuracy of the predictions was quantified as the absolute value of the difference between the prediction and the participant's actual memory score. We used a nonparametric approach to assess whether these predictions were reliably better than chance. We repeated the above process 100,000 times but shuffled participants' behavioral data before running each regression. This provided us with a null distribution of absolute prediction errors, which we used to calculate the 
likelihood of making predictions as accurate as those obtained using our true models. It should be noted that the shuffling procedure used to generate these null models does not influence the intercept term, which reflects the average performance across subjects. Thus, the predictions generated by these models are much more accurate than a model that randomly predicts performance.

GLM for trial-evoked responses. We assessed whether trial-evoked responses in distinct hippocampal subregions (DG/CA3, CA1, and subiculum) were differentially related to encoding and retrieval. After preprocessing, the four functional runs were concatenated in time. The time series in each voxel was modeled using a GLM implemented by $3 \mathrm{dDe}-$ convolve (http://afni.nimh.nih.gov/afni). To assess which regions produced responses that were related to the formation of long-term memories, Encoding Task trials were binned according to long-term memory performance on day 2 [Miss, Item Only+ Low Confidence Association (Item + LC), High Confidence Association (HC)]. Math and Retrieval Task trials were also binned according to accuracy (Correct, Incorrect). All trials were modeled with a $3 \mathrm{~s}$ boxcar that corresponded to the object presentation period, convolved with an HRF. The average $\beta$ estimate for each regressor was then extracted from anatomically defined hippocampal ROIs. Associative encoding success was assessed in a 3 (hippocampal ROI: DG/ CA3, CA1, Sub) $\times 2$ (day 2 accuracy: Item $+\mathrm{LC}, \mathrm{HC})$ repeated-measures ANOVA. Planned follow-up comparisons between memory conditions were also performed in each region. Associative retrieval success was assessed in a 3 (hippocampal ROI: DG/CA3, CA1, Sub) $\times 2$ (day 1 accuracy: Correct, Incorrect) repeated-measures ANOVA. Planned follow-up comparisons between retrieval conditions were also performed in each region. Participants were removed from these analyses if they had fewer than 12 trials in a condition of interest, leaving 16 participants for the Encoding Success analyses and 11 participants for the Retrieval Success analyses.

\section{$\beta$-Series functional connectivity}

Finally, we compared the background connectivity measures described above to more traditional "trial-based" $\beta$-series correlation (BSC) estimates of connectivity, an approach that is more sensitive to fluctuations in trial-evoked BOLD responses across regions (Rissman et al., 2004). Functional data were preprocessed using the steps described in the trialevoked GLM section. To obtain estimates of BOLD responses to individual trials, each functional run was modeled using one GLM that included a separate regressor for each Encoding and Retrieval Task trial ( $3 \mathrm{~s}$ boxcar convolved with HRF). Each model also included regressors that coded for the six motion parameters, linear drift, and Math Task trials $(3 \mathrm{~s}$ boxcar for all trials, convolved with HRF). The average $\beta$ estimates for each trial were then extracted from each ROI and concatenated to form a $\beta$-series vector of Encoding Task trials and a $\beta$-series vector of Retrieval Task trials for each region. We estimated trial-based functional connectivity during each task by correlating the $\beta$-series vectors across different regions and measured the differences in this functional connectivity across the memory tasks. We also determined whether trial-evoked functional connectivity was related to memory accuracy across subjects by correlating memory performance with baseline-corrected connectivity estimates obtained from all Encoding or Retrieval Task trials.

\section{Results}

\section{Behavior}

Overall, participants exhibited high performance across the three experimental tasks (Fig. 2). During the Encoding Task, participants reported successful task performance (i.e., generating a scenario) on $95 \%$ (SD, 4\%) of trials. Importantly, performance on the immediate Retrieval Task was also high. On average, participants correctly recalled the paired object on $80 \%$ (SD, 15\%) of trials. Critically, despite the overall high performance, there was also high variability in memory performance across participants (range, 63-97\% correct), and this was used to assess the relationship between neural measures and performance across participants.

On the long-term memory test $24 \mathrm{~h}$ after the scanned session, participants correctly identified old objects on $90 \%$ (SD, 10\%) of trials of a recognition test and correctly recalled the previously associated object on 65\% (SD, 20\%) of trials (Fig. 2). Trials were also divided according to associative confidence ratings (high, 3 and 4; low, 1 and 2). Low-confidence associative trials had a significantly lower d-prime $\left(d^{\prime}=1.1\right)$ than high-confidence associative trials $\left(d^{\prime}=3.2 ; t_{(16)}=11.4 ; p=4.2 \times 10^{-9}\right)$. The proportion of high-confidence associative trials was also quite variable across participants (range, $30-74 \%$ of old trials), making it well suited for an analysis of individual differences.

\section{fMRI results}

Trial-evoked GLM analysis

We first assessed whether BOLD responses in different hippocampal subregions were related to subsequent memory and 
a DG/CA3

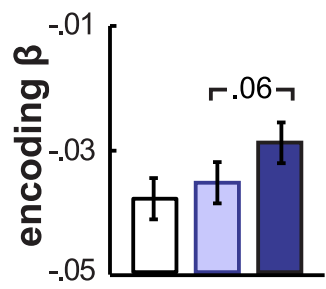

b
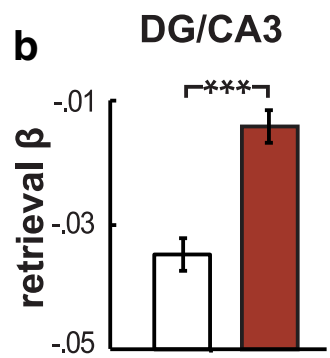

CA1

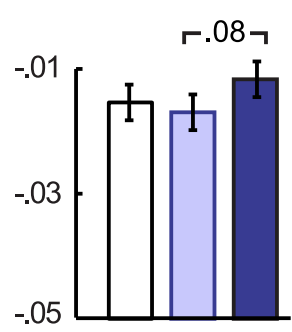

Subiculum

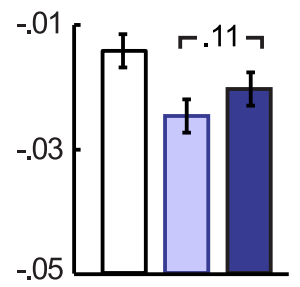

miss

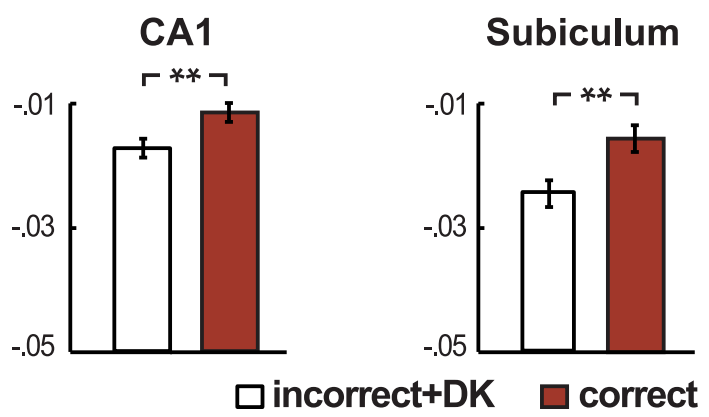

Figure 3. Trial-evoked BOLD responses. $\boldsymbol{a}$, Subsequent memory effects during the Encoding Task. Bars indicate the average $\beta$ estimates from a GLM that estimated the BOLD response to Encoding Task trials binned according to subsequent memory performance on day 2: miss, item memory without correct associative/item memory with low-confident associative memory (item $+\mathrm{LC}$ ), and item memory with high-confident associative memory (HC). A significant main effect of associative memory was found across hippocampal subfields $\left(t_{(15)}=7.87 ; p=0.02\right)$. $\boldsymbol{b}$, Retrieval success effects during the Retrieval Task. Bars indicate the average $\beta$ estimates from a GLM that estimated the BOLD response to Retrieval Task trials binned according to day 1 associative memory performance: correct and incorrect plus don't know (incorrect+DK). A significant main effect of retrieval success was found across hippocampal subfields $\left(t_{(10)}=62.20 ; p=0.0001\right)$. Participants were excluded from both analyses if they had $<12$ trials in a bin. Error bars indicate the SE of the difference score. ${ }^{* *} p<0.01$; $^{* * *} p<0.005$.

retrieval success in this task. Separate repeated-measures ANOVAs were conducted for the Encoding and Retrieval Tasks [En-

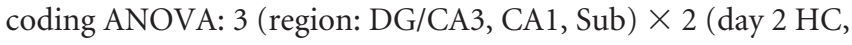
Item + LC); Retrieval ANOVA: 3 (region: DG/CA3, CA1, Sub) $\times$ 2 (day 1 Correct, Incorrect)]. Consistent with prior fMRI studies, hippocampal BOLD activation was related to both successful associative encoding (main effect: $F_{(1,15)}=7.87 ; p=0.02$ ) and successful retrieval (main effect: $F_{(1,10)}=62.20 ; p=0.0001$ ), with greater hippocampal responses elicited on trials where successful associative memories were encoded or retrieved compared with trials where associative memory was unsuccessful (Fig. 3). There was no interaction between subsequent memory and region for the Encoding Task $\left(F_{(1,15)}=0.16 ; p=0.85\right)$, but a significant interaction between memory accuracy and region was found for the Retrieval Task $\left(F_{(1,10)}=10.07 ; p=0.001\right)$. This interaction, which was driven by a stronger retrieval success effect in area DG/CA3 than other hippocampal regions (both $p<0.02$ ), is consistent with the theoretical role of area CA3 in associative reactivation (Marr, 1971; Treves and Rolls, 1992). Together, these effects provide evidence that the hippocampus is involved in the formation and retrieval of associations in this task, but the differentiation between the contributions of individual subregions is less clear from this analytic approach. Moreover, it is well established that these regions are highly interconnected, and this approach does not shed any light on how memory performance may also be influenced by the interaction between regions, the central question of this experiment.
Background connectivity analyses

To assess the relationship between memory performance and hippocampal connectivity, we used a measure of "background" connectivity. Specifically, we measured the correlation in lowfrequency BOLD responses between two regions over whole blocks of the Encoding and Retrieval Tasks after removing taskevoked responses. This type of approach has been successfully applied to reveal changes in connectivity with attention and working memory (Newton et al., 2011; Norman-Haignere et al., 2012) and is further motivated by recent findings that processes occurring both before and after a trial are related to memory success (Tambini et al., 2010; Addante et al., 2011; Ben-Yakov and Dudai, 2011; Fell et al., 2011; Duncan et al., 2012b). After taking several steps to filter out nuisance factors (see Materials and Methods), background connectivity was estimated using time series extracted from anatomical ROIs drawn for individual participants. These regions included the hippocampal subfields (areas DG/CA3, CA1, and subiculum), medial temporal cortical regions (perirhinal and parahippocampal cortices), and midbrain nuclei (VTA and substantia nigra). Data for the entorhinal cortex were excluded from analyses because two-thirds of participants had significant signal dropout and distortions (see Materials and Methods).

Modulation of CA1 background connectivity by memory task Background connectivity between CA1 and DG/CA3 was significantly greater during the Retrieval compared with the Encoding Task $\left(t_{(15)}=3.3 ; p=0.003\right.$; all other regions, $p>0.18$; Fig. 4$)$. To further evaluate the specificity of this relationship, we submitted CA1 connectivity estimates (CA1-DG/CA3, CA1-subiculum, CA1-perirhinal cortex, CA1-parahippocampal cortex, and CA1-VTA) to a repeated-measures ANOVA (Region Pair $\times$ Memory Task). We found a significant interaction between region pair and task $\left(F_{(4,60)}=3.4 ; p=0.02\right)$ but no significant main effect of task $\left(F_{(1,15)}=0.02 ; p=0.89\right)$, indicating that there was not a general tendency for higher background connectivity during the Retrieval Task across all tested ROI pairs. The selective increase in CA1-DG/CA3 background connectivity during the Retrieval compared with Encoding Task is consistent with the potential importance of this pathway for associative retrieval.

Next, an additional control analysis was performed to adjust for the inherent asymmetry in the order of task presentation. Because of the nature of the memory test, the Encoding Task block always occurred first in each run, and the Retrieval Task block was always last. However, since participants performed four alternating blocks of each task, we could adjust for the effects of overall time/order by removing both the first Encoding Task block and last Retrieval Task block. Importantly, we replicated the effect with this adjustment. Namely, CA1-DG/CA3 functional connectivity was still significantly higher during the Re- 
trieval compared with the Encoding Task $\left(t_{(15)}=2.3 ; p=0.04\right.$; Fig. $\left.4 b\right)$. Furthermore, after adjusting for task order, functional connectivity between area CA1 and the perirhinal cortex was significantly higher during the Encoding compared with the Retrieval Task $\left(t_{(15)}=2.27 ; p=\right.$ 0.04 ), consistent with the idea that CA1 input from the medial temporal lobe cortex may be prioritized during encoding (Hasselmo et al., 2002; Colgin and Moser, 2010; Duncan et al., 2012b).

\section{Behavioral correlations}

The above results establish, using a within-subjects analysis, that the overall magnitude of background connectivity between CA1 and related structures is differentially modulated during the Encoding and Retrieval Tasks. To examine whether and how shifts in background connectivity are related to selective encoding and retrieval operations, we next examined whether background connectivity is related to encoding and retrieval success across subjects.

We found that background connectivity between area CA1 and the VTA during the Encoding Task significantly correlated with subsequent long-term associative memory tested on day $2\left(r_{(13)}=0.64 ; p=\right.$ 0.02 ; Fig. $5 a)$, but not with immediate memory $(r=15 ; p=0.62)$. This relationship held after down-weighting the influence of extreme points using robust regression $(\beta=0.09 ; p=0.03)$. Importantly, background connectivity between other CA1 pairs was not related to day 2 memory performance (all $p>0.32$ for other CA1 pairs). Interestingly, CA1VTA connectivity during the Encoding Task still predicted day 2 memory when controlling for day 1 associative memory, using a partial correlation $\left(r_{(13)}=0.74 ; p=0.006\right)$. Together, these results provide strong evidence that, across subjects, the variability in VTA-CA1 background connectivity during encoding is uniquely related to successful long-term memory formation.

In contrast, connectivity between CA1 and DG/CA3 during the Retrieval Task was significantly related to retrieval success measured on day $1\left(r_{(13)}=0.60 ; p=0.03\right.$; Fig. $\left.5 b\right)$. Importantly, this was the only CA1 region pair that significantly predicted retrieval success (all $p>0.25$ for CA1 pairs). The reliability of the relationship between CA1-DG/CA3 functional connectivity and retrieval success was also confirmed using robust regression $(\beta=$ $0.06 ; p=0.04)$.

Thus, together, we found that background connectivity between areas CA1 and DG/CA3 was significantly related to successful retrieval, but not encoding, whereas CA1-VTA functional connectivity was related to successful long-term encoding, but not retrieval. To further test the reliability of this double dissociation, we performed a nonparametric-shuffle interaction test: we randomly shuffled the behavioral data and recalculated the correlations for both region pairs and then calculated the interaction term [(CA1\&DG/CA3 retrieval correlation -
CA1\&DG/CA3 encoding correlation $)]-[(\mathrm{CA} 1 \& V T A$ retrieval correlation - CA1\&VTA encoding correlation)] 100,000 times to obtain a distribution of the null hypothesis. Using this approach, we calculated the probability of finding an interaction term as large as was observed in the dataset. We found that the probability was below 5\% (two-tailed $p=0.016$; Fig. $5 c$ ). Additional shuffle tests were performed to determine the driving force behind the interaction, and we found marginally significant effects for the difference between encoding and retrieval for both CA1-DG/CA3 correlations (one-tailed $p=0.04$ ) and CA1-VTA correlations (one-tailed $p=$ 0.04). Together, these interactions further underscore the finding that differential background connectivity with CA1 is related to successful retrieval and encoding, respectively.

Finally, we used a cross-validation procedure to assess how well these results might generalize to a larger population. To do this, we systematically removed each participant, estimated the relationship between CA1 connectivity and behavior in the remaining participants using a regression model, and used the results of that model to predict memory performance for the removed participant based on his or her CA1 connectivity. The accuracy of the prediction was quantified by comparing the participant's actual performance to his or her predicted performance. For example, if a participant had a corrected associative 
a

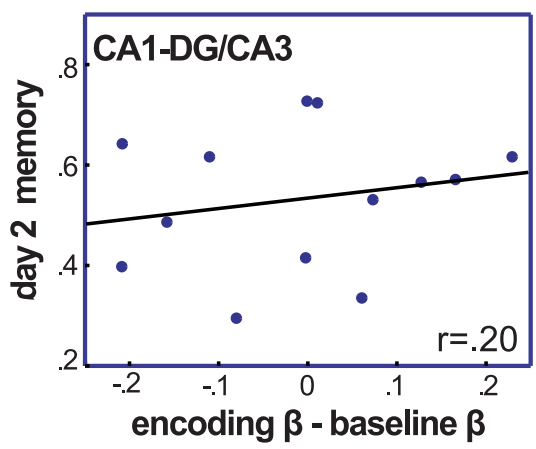

b

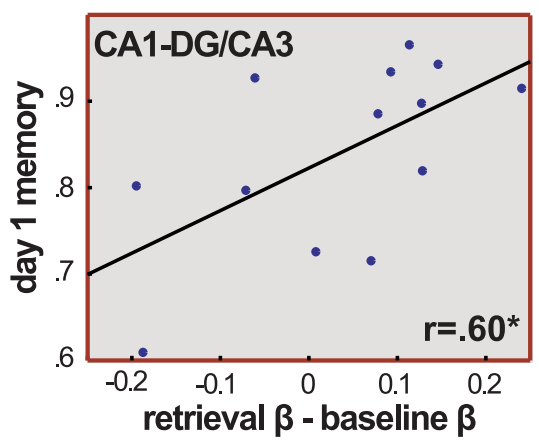

C

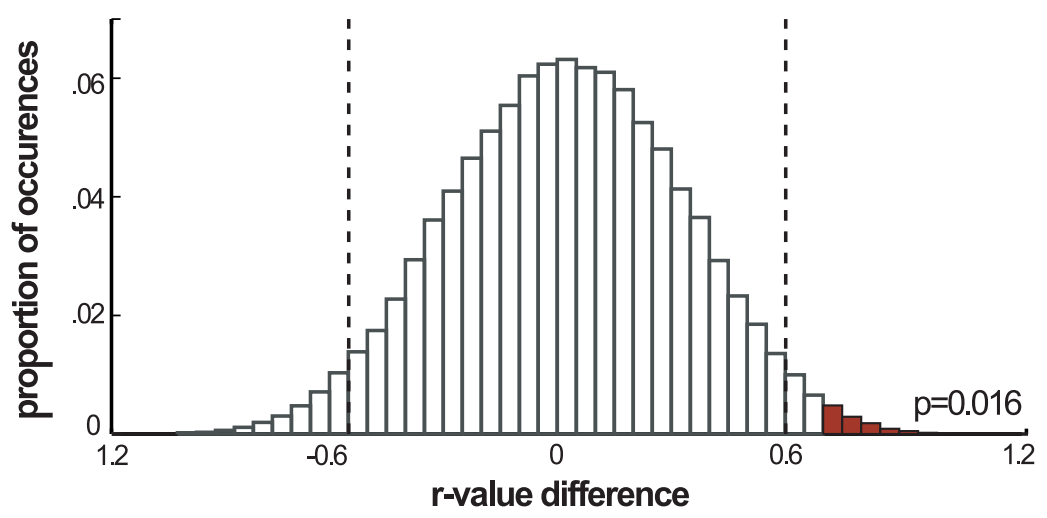

Figure 5. Memory performance and changes in functional connectivity. $\boldsymbol{a}$, Corrected day 2 high-confidence associative memory is plotted against Encoding Task functional connectivity (Encoding - Baseline Math). CA1-VTA encoding functional connectivity was significantly correlated with subsequent memory performance $(p=0.02) \cdot \boldsymbol{b}$, Corrected day 1 associative memory is plotted against Retrieval Task functional connectivity (Retrieval - Baseline Math). CA1-DG/CA3 retrieval functional connectivity was significantly correlated with memory performance $(p=0.03)$. $c$, The proportion of randomly shuffled data that resulted in interaction terms of different magnitudes. Vertical dashed lines indicate the significance threshold of this nonparametric test at the $\alpha=0.05$ level, and red bars mark shuffles that resulted in an interaction term with a larger value than that which was obtained in the dataset. Two-tailed $p=0.016$.

memory score of $60 \%$ and the model predicted that his or her performance would be $67 \%$, the error rate would be $7 \%$. Using this approach, we were able to predict participants' day 2 corrected associative memory performance based on their CA1VTA functional connectivity during the Encoding Task with an average error rate of $9.1 \%$. CA1-DG/CA3 functional connectivity during the Retrieval Task was a similarly good predictor of retrieval success on day 1 , with an error rate of $8.4 \%$. To assess whether these predictions were reliably better than chance, we used a nonparametric procedure in which the process was repeated 100,000 times, shuffling behavioral performance on each iteration. We found that both predictions were reliably more accurate than their corresponding null models (CA1-VTA Encoding Task: null error rate of $12.3 \%$, $p=0.001$; CA1-DG/CA3 Retrieval Task: null error rate of $9.7 \%, p=0.03$ ). Importantly, CA1-VTA Retrieval Task connectivity was not a reliable predictor of day 1 memory success $(p=0.76)$, and CA1DG/CA3 Encoding Task connectivity was not a reliable predictor of day 2 associative memory ( $p=0.27)$. These results demonstrate that the relationships between CA1 connectivity and memory performance observed in this dataset are robust enough to generate reliable predictions in a broader population.

\section{$\boldsymbol{\beta}$-Series correlation analysis}

Whereas the current experiment was specifically designed to assess changes in lowfrequency background connectivity, we also directly examined whether these results were driven by trial-evoked responses. Specifically, we conducted a BSC analysis (Rissman et al., 2004), an approach that computes the correlation in trial-evoked BOLD responses across all trials in a particular condition. We reasoned that, if the background connectivity results reported above were primarily driven by trial-evoked activity, we should see parallel results in the BSC analysis. This, however, was not the case. Specifically, CA1-DG/CA3 BSC did not differ between the Retrieval and Encoding Tasks $\left(t_{(15)}=-0.74 ; p=0.47\right)$. We also used robust regression to predict associative memory performance across subjects (a parallel analysis to the background connectivity approach) based on trial-evoked CA1 functional connectivity and found that CA1-VTA BSC during the Encoding Task was not a reliable predictor of long-term memory performance $(\beta=0.04 ; p=0.36)$, nor did CA1-DG/CA3 BSC during the Retrieval Task reliably predict retrieval success $(\beta=0.04 ; p=0.18)$.

Finally, we directly compared how well background connectivity and trial-evoked connectivity (BSC) predicted memory performance by including both measures in the same robust regression models. The standardized $\beta$ estimates of background connectivity were numerically larger than the standardized $\beta$ estimates for trialevoked connectivity in both the encoding and retrieval models (Table 1). Together, these results confirm that the predictive capacity of the background connectivity analyses was, indeed, not solely driven by BOLD responses evoked by the trials themselves.

\section{Discussion}

We used high-resolution fMRI to measure background functional connectivity with hippocampal area CA1 during blocks of associative encoding and retrieval. Using this approach, we iden- 
Table 1. Comparison of state-based background and $\beta$-series functional connectivity

\begin{tabular}{lll}
\hline & Standardized $\beta$ & $p$ value \\
\hline Encoding task & & \\
Background & $0.59(0.30)$ & 0.07 \\
$\beta$ series & $0.11(0.30)$ & 0.71 \\
Retrieval task & & \\
Background & $0.56(0.38)$ & 0.17 \\
$\beta$ series & $0.05(0.38)$ & 0.89 \\
\hline
\end{tabular}

Background and $\beta$-series measures of functional connectivity were used to predict memory performance across participants in the same robust regression models. Connectivity during the Encoding Task was used to predict long-term associative memory performance (day 2), and connectivity during the Retrieval Task was used to predict day 1 memory performance. Standardized $\beta$ estimates reflect how well each measure predicted memory performance, adjusting for the predictive power of the other. SE of the estimate is included in parentheses.

tified a dissociation between the CA1 pathways that are related to episodic encoding and retrieval. Specifically, we found that CA1CA3/DG background connectivity is greater during associative retrieval compared with encoding and, across subjects, relates to retrieval success. Conversely, CA1-VTA connectivity during encoding, but not retrieval, is related to long-term, but not immediate, memory success across subjects. Together, these results offer strong support for the hypothesis that hippocampal subregion CA1 plays an important role in biasing hippocampal processing toward encoding or retrieval operations.

Importantly, these results provide leverage to theoretical models proposing that successful encoding and retrieval are related to processing along distinct CA1 pathways (Hasselmo et al., 2002; Colgin and Moser, 2010). Specifically, because area CA1 may receive information about retrieved associations and events occurring in the environment from distinct pathways, changes in the relative strength of these inputs could bias hippocampal processing and output toward retrieving previously learned associations or encoding new ones. Furthermore, CA1 synaptic plasticity is dopamine dependent (Frey et al., 1990; Huang and Kandel, 1995; Li et al., 2003), suggesting that increased functional interactions between this region and areas providing dopaminergic input could facilitate the formation of long-lasting memories (Lisman and Grace, 2005).

To test these hypotheses, we adopted an innovative approach that allowed us to relate functional connectivity and behavior: specifically, we measured the relationship between low-frequency fluctuations in the BOLD response across entire task blocks rather than limiting our analysis to trial-evoked BOLD responses. This approach was motivated by recent findings that memory success is related to neural processing occurring before and after encoding or retrieval opportunities (Tambini et al., 2010; Addante et al., 2011; Ben-Yakov and Dudai, 2011; Fell et al., 2011), potentially reflecting preparatory modes or postencoding processing. Moreover, recent behavioral experiments in humans have identified lingering mnemonic biases by demonstrating, for example, that encoding novel events and retrieving familiar events facilitates the subsequent encoding or retrieval processes, respectively (Duncan et al., 2012b; Fenker et al., 2008). The present findings add to this literature by showing that background connectivity predicts memory performance better than measures of trialevoked connectivity, highlighting the potential mnemonic importance of processes that extend beyond the temporal boundaries of individual trials.

Using this approach, we found that CA1-VTA background connectivity during encoding specifically predicted individual differences in subsequent long-term memory. Although it is unknown in what manner VTA BOLD activation is related to dopa- mine function, this result nicely parallels findings in rodents showing that late-phase LTP in area CA1 is dopamine dependent (Frey et al., 1990; Huang and Kandel, 1995; Li et al., 2003). Moreover, these results are broadly consistent with theoretical work (Lisman and Grace, 2005), anatomical connectivity (Gasbarri et al., 1994), and animal studies (Frey et al., 1990; Huang and Kandel, 1995; Li et al., 2003) predicting that VTA projections to area CA1 are critical for plasticity that supports long-term memory encoding. It should be noted, however, that functional connectivity does not indicate directionality, leaving open the possibility that indirect projections from area CA1 to the VTA may also contribute to the observed results. Interestingly, activation of this indirect pathway results in increased dopaminergic release in area CA1 (Lisman and Grace, 2005). Thus, increased connectivity along either route could theoretically increase dopamine release to area CA1. Future pharmacological work is, however, necessary to determine whether and how the mechanism underlying these findings is related to dopamine transmission. Nonetheless, the results reported here strongly implicate VTA connectivity with CA1 during successful long-term memory formation.

This study also demonstrates that CA1-DG/CA3 functional connectivity is related to successful retrieval. This result converges with electrophysiological data from rodents (Montgomery and Buzsaki, 2007) showing greater coherence between local field potentials generated by areas CA3 and CA1 when rats paused at decision points on a maze, potentially reflecting the retrieval of past trial outcomes. Additionally, synchronous firing between areas CA3 and CA1 has been related to replay during sharp wave ripples (Carr et al., 2012). Although this previous work demonstrates that CA1-CA3 connectivity increases during periods when retrieval may be more likely to occur, our results link this effect directly to memory retrieval by demonstrating that functional connectivity between DG/CA3 and area CA1 correlates with retrieval success. Moreover, we found that trial-evoked responses in DG/CA3 were especially related to retrieval success, consistent with the theoretical role of area CA3 in associative retrieval (Treves and Rolls, 1992). Together, these findings suggest that the propagation of associations reactivated within the CA3 network through area CA1 before reaching cortical regions supports associative retrieval. It should also be noted, however, that the comparatively lower connectivity between these areas during encoding does not imply that input from areas DG/CA3 is not necessary or important for encoding. In fact, the plasticity of Schaffer collateral synapses is thought to support associative memory formation (Huang and Kandel, 1995). Additionally, the reversible inhibition of input from area CA3 disrupts both recall and rapid one-trial contextual learning (Nakashiba et al., 2008). Instead, our results simply suggest that correlated activation between DG/CA3 and CA1 is stronger during successful retrieval relative to encoding.

We also found that the strength of CA1-DG/CA3 functional connectivity differed between the Encoding and Retrieval Tasks, a result that may speak to the hypothesis that episodic encoding and retrieval are associated with different processing states. Specifically, increased CA3 input to area CA1 may bias the system toward recalling associated memories, whereas shifts toward medial temporal cortical input, containing information about the current environment, may favor the formation of distinct memories (Hasselmo et al., 2002; Colgin and Moser, 2010; Duncan et al., 2012b; Easton et al., 2012). Extended periods of episodic encoding and retrieval could, thus, be differentially associated with processing in these CA1 pathways. Broadly consistent with this framework, CA1 connectivity with perirhinal and 
parahippocampal cortices was numerically greater during the Encoding than the Retrieval Task, with the difference reaching significance for the perirhinal cortex when adjusting for the order of tasks. We were not able to assess CA1 connectivity with the entorhinal cortex, which factors more prominently into the above models, however, because of substantial signal dropout and distortions.

Critically, functional connectivity results reported here were interpreted with respect to memory performance, circumventing potential limitations in their interpretation. For example, with its close proximity to vasculature, the VTA is particularly susceptible to physiological noise, which can artificially inflate estimates of functional connectivity. However, because we measured differences in connectivity between baseline and memory blocks, any biases attributable to physiological noise would be removed since this signal should be consistently present in all blocks. More importantly, VTA connectivity did not show simple task-related changes in the strength of connectivity. Instead, changes in VTACA1 connectivity were specifically related to individual differences in memory performance, a pattern that is hard to relate to simple physiological noise. Similarly, we also see that functional connectivity between DG/CA3 and CA1 is related to behavioral measures of successful retrieval. Although we attempted to limit the differences between the Encoding and Retrieval Tasks, unavoidable differences, such as perceptual novelty, could serve as alternative explanations for increased DG/CA3-CA1 connectivity during the Retrieval Task. However, the finding that this increase in connectivity predicted retrieval performance strongly favors the interpretation that functional connectivity along this pathway is related to retrieval.

More broadly, this study serves as an example of how the development of high-resolution fMRI has served as a critical bridge between hippocampal research performed in humans and animals (Carr et al., 2010). In recent years, high-resolution hippocampal imaging has been used to determine how BOLD activation in distinct hippocampal subregions is related to episodic encoding and retrieval (Zeineh et al., 2003; Eldridge et al., 2005; Preston et al., 2010), computational processes such as pattern separation and novelty detection (Kirwan and Stark, 2007; Bakker et al., 2008; Chen et al., 2011; Duncan et al., 2012a; Lacy et al., 2011), and reward modulation of memory (Wolosin et al., 2012). The current study builds off of this foundation to investigate a question that had previously only been explored in animal models: How do interactions between hippocampal subregions differentially support memory encoding and retrieval? Highresolution fMRI allowed us to simultaneously record responses from distinct hippocampal subregions and relate the correlations between these signals to basic human memory processes.

In conclusion, by measuring changes in background functional connectivity within the medial temporal lobe and midbrain, we revealed a potential role for area CA1 in directing hippocampal processing toward encoding or retrieving memories. Heightened connectivity between this subregion and the VTA predicted successful long-term memory formation, whereas heightened connectivity between area CA1 and the DG/CA3 subregions predicted successful associative retrieval. These results connect predictions derived from computational models and basic neuroscience research to the human brain. They additionally highlight the potential of background connectivity analyses to reveal theoretically important processes that extend beyond the limits of trials.

\section{References}

Addante RJ, Watrous AJ, Yonelinas AP, Ekstrom AD, Ranganath C (2011) Prestimulus theta activity predicts correct source memory retrieval. Proc Natl Acad Sci U S A 108:10702-10707. CrossRef Medline

Bakker A, Kirwan CB, Miller M, Stark CE (2008) Pattern separation in the human hippocampal CA3 and dentate gyrus. Science 319:1640-1642. CrossRef Medline

Ben-Yakov A, Dudai Y (2011) Constructing realistic engrams: poststimulus activity of hippocampus and dorsal striatum predicts subsequent episodic memory. J Neurosci 31:9032-9042. CrossRef Medline

Bethus I, Tse D, Morris RG (2010) Dopamine and memory: modulation of the persistence of memory for novel hippocampal NMDA receptordependent paired associates. J Neurosci 30:1610-1618. CrossRef Medline

Birn RM, Diamond JB, Smith MA, Bandettini PA (2006) Separating respiratory-variation-related fluctuations from neuronal-activity-related fluctuations in fMRI. Neuroimage 31:1536-1548. CrossRef Medline

Burock MA, Buckner RL, Woldorff MG, Rosen BR, Dale AM (1998) Randomized event-related experimental designs allow for extremely rapid presentation rates using functional MRI. Neuroreport 9:3735-3739. CrossRef Medline

Carr VA, Rissman J, Wagner AD (2010) Imaging the human medial temporal lobe with high-resolution fMRI. Neuron 65:298-308. CrossRef Medline

Carr MF, Karlsson MP, Frank LM (2012) Transient slow gamma synchrony underlies hippocampal memory replay. Neuron 75:700-713. CrossRef Medline

Chen J, Olsen RK, Preston AR, Glover GH, Wagner AD (2011) Associative retrieval processes in the human medial temporal lobe: hippocampal retrieval success and CA1 mismatch detection. Learn Mem 18:523-528. CrossRef Medline

Colgin LL, Moser EI (2010) Gamma oscillations in the hippocampus. Physiology (Bethesda) 25:319-329. CrossRef Medline

Cordes D, Haughton VM, Arfanakis K, Carew JD, Turski PA, Moritz CH, Quigley MA, Meyerand ME (2001) Frequencies contributing to functional connectivity in the cerebral cortex in "resting-state" data. AJNR Am J Neuroradiol 22:1326-1333. Medline

D'Ardenne K, McClure SM, Nystrom LE, Cohen JD (2008) BOLD responses reflecting dopaminergic signals in the human ventral tegmental area. Science 319:1264-1267. CrossRef Medline

Duncan K, Ketz N, Inati SJ, Davachi L (2012a) Evidence for area CA1 as a match/mismatch detector: a high-resolution fMRI study of the human hippocampus. Hippocampus 22:389-398. CrossRef Medline

Duncan K, Sadanand A, Davachi L (2012b) Memory's penumbra: episodic memory decisions induce lingering mnemonic biases. Science 337:485487. CrossRef Medline

Duvernoy HM (2005) The human hippocampus: functional anatomy, vascularization, and serial sections with MRI. New York: Springer.

Easton A, Douchamps V, Eacott M, Lever C (2012) A specific role for septohippocampal acetylcholine in memory? Neuropsychologia 50:31563168. CrossRef Medline

Eichenbaum H, Yonelinas AP, Ranganath C (2007) The medial temporal lobe and recognition memory. Annu Rev Neurosci 30:123-152. CrossRef Medline

Eldridge LL, Engel SA, Zeineh MM, Bookheimer SY, Knowlton BJ (2005) A dissociation of encoding and retrieval processes in the human hippocampus. J Neurosci 25:3280-3286. CrossRef Medline

Fell J, Ludowig E, Staresina BP, Wagner T, Kranz T, Elger CE, Axmacher N (2011) Medial temporal theta/alpha power enhancement precedes successful memory encoding: evidence based on intracranial EEG. J Neurosci 31:5392-5397. CrossRef Medline

Fenker DB, Frey JU, Schuetze H, Heipertz D, Heinze HJ, Duzel E (2008) Novel scenes improve recollection and recall of words. J Cogn Neurosci 20:1250-1265. CrossRef Medline

Frey U, Schroeder H, Matthies H (1990) Dopaminergic antagonists prevent long-term maintenance of posttetanic LTP in the CA1 region of rat hippocampal slices. Brain Res 522:69-75. CrossRef Medline

Gasbarri A, Packard MG, Campana E, Pacitti C (1994) Anterograde and retrograde tracing of projections from the ventral tegmental area to the hippocampal formation in the rat. Brain Res Bull 33:445-452. CrossRef Medline

Gasbarri A, Sulli A, Packard MG (1997) The dopaminergic mesencephalic 
projections to the hippocampal formation in the rat. Prog Neuropsychopharmacol Biol Psychiatry 21:1-22. CrossRef Medline

Giraud P, Kantor G, Yassa M, Zefkili S, Dejean C, Lisbona A, Campana F, Mahé MA (2011) Two-year clinical experience with tomotherapy: the French national cancer institute project on implementing new technology. Cancer Invest 29:557-563. CrossRef Medline

Hasselmo ME, Bodelón C, Wyble BP (2002) A proposed function for hippocampal theta rhythm: separate phases of encoding and retrieval enhance reversal of prior learning. Neural Comput 14:793-817. CrossRef Medline

Huang YY, Kandel ER (1995) D1/D5 receptor agonists induce a protein synthesis-dependent late potentiation in the CA1 region of the hippocampus. Proc Natl Acad Sci U S A 92:2446-2450. CrossRef Medline

Insausti R, Juottonen K, Soininen H, Insausti AM, Partanen K, Vainio P, Laakso MP, Pitkänen A (1998) MR volumetric analysis of the human entorhinal, perirhinal, and temporopolar cortices. AJNR Am J Neuroradiol 19:659-671. Medline

Kirwan CB, Stark CE (2007) Overcoming interference: an fMRI investigation of pattern separation in the medial temporal lobe. Learn Mem 14: 625-633. CrossRef Medline

Kirwan CB, Jones CK, Miller MI, Stark CE (2007) High-resolution fMRI investigation of the medial temporal lobe. Hum Brain Mapp 28:959-966. CrossRef Medline

Lacy JW, Yassa MA, Stark SM, Muftuler LT, Stark CE (2011) Distinct pattern separation related transfer functions in human CA3/dentate and CA1 revealed using high-resolution fMRI and variable mnemonic similarity. Learn Mem 18:15-18. CrossRef Medline

Li S, Cullen WK, Anwyl R, Rowan MJ (2003) Dopamine-dependent facilitation of LTP induction in hippocampal CA1 by exposure to spatial novelty. Nat Neurosci 6:526-531. CrossRef Medline

Lisman JE, Grace AA (2005) The hippocampal-VTA loop: controlling the entry of information into long-term memory. Neuron 46:703-713. CrossRef Medline

Marr D (1971) Simple memory: a theory for archicortex. Philos Trans R Soc Lond B Biol Sci 262:23-81. CrossRef Medline

McClelland JL, McNaughton BL, O’Reilly RC (1995) Why there are complementary learning systems in the hippocampus and neocortex: insights from the successes and failures of connectionist models of learning and memory. Psychol Rev 102:419-457. CrossRef Medline

Meeter M, Shohamy D, Myers CE (2009) Acquired equivalence changes stimulus representations. J Exp Anal Behav 91:127-141. CrossRef Medline

Montgomery SM, Buzsáki G (2007) Gamma oscillations dynamically couple hippocampal CA3 and CA1 regions during memory task performance. Proc Natl Acad Sci U S A 104:14495-14500. CrossRef Medline

Nakashiba T, Young JZ, McHugh TJ, Buhl DL, Tonegawa S (2008) Transgenic inhibition of synaptic transmission reveals role of CA3 output in hippocampal learning. Science 319:1260-1264. CrossRef Medline

Newton AT, Morgan VL, Rogers BP, Gore JC (2011) Modulation of steady state functional connectivity in the default mode and working memory networks by cognitive load. Hum Brain Mapp 32:1649-1659. CrossRef Medline

Norman-Haignere SV, McCarthy G, Chun MM, Turk-Browne NB (2012) Category-selective background connectivity in ventral visual cortex. Cereb Cortex 22:391-402. CrossRef Medline

O'Carroll CM, Martin SJ, Sandin J, Frenguelli B, Morris RG (2006) Dopaminergic modulation of the persistence of one-trial hippocampusdependent memory. Learn Mem 13:760-769. CrossRef Medline

Ojemann JG, Akbudak E, Snyder AZ, McKinstry RC, Raichle ME, Conturo TE (1997) Anatomic localization and quantitative analysis of gradient refocused echo-planar fMRI susceptibility artifacts. Neuroimage 6:156167. CrossRef Medline

Olman CA, Davachi L, Inati S (2009) Distortion and signal loss in medial temporal lobe. PLoS One 4:e8160. CrossRef Medline

O’Reilly RC, McClelland JL (1994) Hippocampal conjunctive encoding, storage, and recall: avoiding a trade-off. Hippocampus 4:661-682. CrossRef Medline

Preston AR, Bornstein AM, Hutchinson JB, Gaare ME, Glover GH, Wagner AD (2010) High-resolution fMRI of content-sensitive subsequent memory responses in human medial temporal lobe. J Cogn Neurosci 22:156-173. CrossRef Medline

Pruessner JC, Köhler S, Crane J, Pruessner M, Lord C, Byrne A, Kabani N, Collins DL, Evans AC (2002) Volumetry of temporopolar, perirhinal, entorhinal and parahippocampal cortex from high-resolution MR images: considering the variability of the collateral sulcus. Cereb Cortex 12:1342-1353. CrossRef Medline

Rissman J, Gazzaley A, D'Esposito M (2004) Measuring functional connectivity during distinct stages of a cognitive task. Neuroimage 23:752-763. CrossRef Medline

Scoville WB, Milner B (1957) Loss of recent memory after bilateral hippocampal lesions. J Neurol Neurosurg Psychiatry 20:11-21. CrossRef Medline

Squire LR, Wixted JT, Clark RE (2007) Recognition memory and the medial temporal lobe: a new perspective. Nat Rev Neurosci 8:872-883. CrossRef Medline

Tambini A, Ketz N, Davachi L (2010) Enhanced brain correlations during rest are related to memory for recent experiences. Neuron 65:280-290. CrossRef Medline

Treves A, Rolls ET (1992) Computational constraints suggest the need for two distinct input systems to the hippocampal CA3 network. Hippocampus 2:189-199. CrossRef Medline

Van Dijk KR, Hedden T, Venkataraman A, Evans KC, Lazar SW, Buckner RL (2010) Intrinsic functional connectivity as a tool for human connectomics: theory, properties, and optimization. J Neurophysiol 103:297-321. CrossRef Medline

Wolosin SM, Zeithamova D, Preston AR (2012) Reward modulation of hippocampal subfield activation during successful associative encoding and retrieval. J Cogn Neurosci 24:1532-1547. CrossRef Medline

Zeineh MM, Engel SA, Thompson PM, Bookheimer SY (2003) Dynamics of the hippocampus during encoding and retrieval of face-name pairs. Science 299:577-580. CrossRef Medline 